\title{
MULTI DRUG RESISTANCE IN METHICILLIN RESISTANT STAPHYLOCOCCUS AUREUS (MRSA) ISOLATES FROM A UNIVERSITY HOSPITAL OF WESTERN INDIA.
}

Ravi Gupta, Sandeep Nanda, Sonia Barve

1. Senior Demonstrator, Department of Microbiology, S.N. Medical College, Jodhpur.

2. Assistant Professor, Department of Microbiology, Medical College, Baroda.

3. Associate Professor, Department of Microbiology, Medical College, Baroda.

\author{
CORRESPONDING AUTHOR \\ Ravi Gupta, \\ Senior Demonstrator, \\ S. N. Medical College, Jodhpur, \\ 65/94, Pratap Nagar, Sheopur Road, \\ Sanganer,Jaipur-302033, \\ E-mail: guptabmc@gmail.com, \\ Ph: 00919460031891.
}

ABSTRACT: BACKGROUND: Methicillin Resistant Staphylococcus aureus (MRSA) has emerged as one of the most important nosocomial pathogen. Its remarkable ability to develop resistance to a variety of antibiotics makes it a major threat to public health. OBJECTIVES: - To detect the prevalence and in vitro antimicrobial susceptibility pattern of the MRSA isolates. MATERIALS \& METHODS: - 202 Staphylococcus aureus strains isolated from clinical samples like blood, pus, sputum \& body fluids were screened for Methicillin resistance by standard disk diffusion method and then confirmed with Oxacillin Screening agar. Antibiotic susceptibility of both the MRSA and Methicillin sensitive Staphylococcus aureus (MSSA) for other antibiotics was subsequently carried out by standard disc diffusion method. RESULTS: Methicillin resistance was detected in 114 strains of Staphylococcus aureus giving a prevalence rate of $56.44 \%$. More than $80 \%$ of the MRSA isolates were resistant to antibiotics such as Penicillins, Cephalosporins, Aminoglycosides, Macrolides and Quinolones. All strains were uniformly sensitive to Vancomycin \& Linezolid. Coexisting resistance to most of the antibiotics was significantly higher in the MRSA isolates as compared to the MSSA (Methicillin sensitive Staphylococcus aureus) isolates ( $\mathrm{p}<0.001$ ). INTERPRETATION \& CONCLUSIONS: Multi drug resistance among the MRSA isolates poses a major hurdle in treating systemic infections. Monitoring antibiotic sensitivity pattern, implementing aggressive surveillance measures \& good infection control practices would be helpful in reducing the prevalence of MRSA and its multi drug resistance.

KEYWORDS: Methicillin resistant Staphylococcus aureus (MRSA), Multi drug resistance (MDR).

INTRODUCTION: Staphylococcus aureus (S. aureus) has been increasingly implicated in serious, hospital as well as community acquired infections. This pathogen has been particularly efficient at developing resistance to antimicrobial agents, hence, the antimicrobial chemotherapy for these spp. has always been empirical.1,2 $\mathrm{S}$. aureus strains resistant to Penicillinase-stable $\beta$-lactam antibiotics also known as Methicillin resistant Staphylococcus aureus (MRSA) strains were first isolated from Europe in 1961 and continue to be a major cause of serious infection in humans. ${ }^{3,4}$ In 1982 epidemic MRSA strains (EMRSA) were identified as multi resistant organisms with special capacity to colonize patients and staff causing wide 
spread outbreaks of infections. These epidemic MRSA strains have subsequently spread to various parts of the world leading to epidemic infections of serious proportions. ${ }^{5}$ High prevalence of MRSA in referral hospitals have also been reported from India and neighbouring countries. ${ }^{6-12}$

Concern about MRSA is related not only to its potential for nosocomial transmission but also to its increasing resistance to the commonly used antibiotics like Penicillins, Cephalosporins, Aminoglycosides, Macrolides, and Quinolones. ${ }^{13}$ Emergence of low level resistance to Vancomycin has also been reported. ${ }^{14}$ With limited treatment options available, a knowledge of the prevalence of MRSA and its antimicrobial profile becomes imperative, to select an appropriate empirical treatment for these infections. This formed the basis for carrying out the present study in a tertiary referral University Hospital in Central Gujarat.

MATERIALS AND METHODS: A total of 202 S.aureus strains isolated from various clinical specimens such as blood, pus, sputum \& other body fluids during the period June - December 2005 were included in the study. All the strains were identified by standard techniques. ${ }^{15}$ Each strain was screened for Oxacillin resistance by standard disk diffusion method, using Oxacillin disk $(1 \mu \mathrm{g})$ [Hi-media laboratories. Pvt. Ltd. Mumbai] as per NCCLS recommendation. ${ }^{16} \mathrm{~A}$ zone of inhibition less than $10 \mathrm{~mm}$ or any discernible growth within the zone of inhibition was indicative of methicillin resistance. All methicillin resistant strains were also confirmed by Oxacillin salt-agar screening-plate using $6 \mu \mathrm{g}$ Oxacillin/ml in Muller Hinton agar supplemented with $\mathrm{NaCl}(4 \% \mathrm{w} / \mathrm{v})$. Plates were incubated at $35^{\circ} \mathrm{C}$ and strains showing growth on this medium were taken as MRSA. ${ }^{17}$

Antibiotic sensitivity testing was performed on all isolates by modified Kirby

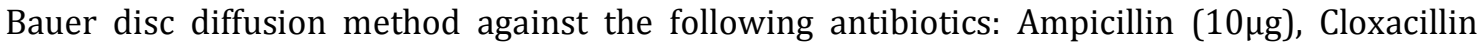
$(1 \mu \mathrm{g})$, Amoxyclav $(10 \mu \mathrm{g})$, Chloramphenicol $(30 \mu \mathrm{g})$, Co-trimoxazole $(25 \mu \mathrm{g})$, Cefotaxime $(30 \mu \mathrm{g})$, Gentamicin $(10 \mu \mathrm{g})$, Norfloxacin $(10 \mu \mathrm{g})$, Ciprofloxacin $(5 \mu \mathrm{g})$, Ofloxacin $(5 \mu \mathrm{g})$, Erythromycin $(15 \mu \mathrm{g})$, Tetracycline $(30 \mu \mathrm{g})$, Vancomycin $(30 \mu \mathrm{g})$ and Linezolid $(30 \mu \mathrm{g})$ [Hi-media laboratories. Pvt. Ltd. Mumbai]. Results were analysed as per recommendation of NCCLS. S. aureus ATCC 25923 was used as the control strain.

Statistical analysis was performed using chi square test $\& Z$ test. A 'p' value of $<0.05$ was considered as statistically significant.

RESULTS: Out of the total 202 isolates of S.aureus, 114 were MRSA (56.44\%). The isolation of MRSA from different clinical specimens is shown in (Table-1).The prevalence of MRSA was significantly different among various clinical specimens. Blood cultures yielded the maximum number of MRSA (50.88\%) followed by pus and wound (32.46\%). Antimicrobial resistance pattern of both the MRSA \& MSSA isolates is depicted in (Figure-1). A high level of resistance ranging from $67.54 \%$ to $100 \%$ was observed among the MRSA isolates to most of the antibiotics but a comparatively low resistance was seen to Chloramphenicol (53.51\%).All the MRSA isolates were resistant to Ampicillin and Cefotaxime (100\%). A marked difference in the antibiotic susceptibility pattern was also observed between the MRSA \& MSSA isolates. Coexisting resistance to most of the antibiotics was significantly higher in the MRSA isolates as compared to the MSSA isolates ( $\mathrm{p}<0.001$ ) (Figure-1). All the S.aureus isolates (MRSA \& MSSA) were uniformly sensitive to Vancomycin and Linezolid. 
DISCUSSION: The present study highlights the problem of MRSA \& its associated multi drug resistance. In India, the importance of MRSA as a problem has been recognized relatively late. ${ }^{6}$ According to a recent Indian study, the MRSA prevalence has increased from 12\% in 1992 to $80.83 \%$ in 1999.10 To add to the problem, multi drug resistance in MRSA has also been documented from various studies across India and is steadily rising 6-12. The alarmingly high prevalence of MRSA (56.44\%) in our study is comparable to the reports from across the country which vary from $31.1 \%$ to $80.83 \% .^{6-11}$ This high prevalence could be attributed to several factors like irrational use of multiple antibiotics, prolonged hospital stay, nasal carriage of MRSA, lack of awareness among the hospital staff and ineffective control measures.

Maximal isolation of the MRSA was from the blood samples unlike other studies where throat swabs and wound swabs were the main source.6, ${ }^{9}$ The antibiotic sensitivity results showed that all the MRSA isolates in our study were significantly more resistant to antibiotics as compared to the MSSA isolates ( $\mathrm{p}<0.05$ to $<0.001$ ). A majority of our MRSA isolates were also resistant to multiple antimicrobial agents, exhibiting a high degree of resistance ranging from $85 \%$ to $100 \%$ to all the major groups of i.e. $\beta$ lactams, Aminoglycosides and Quinolones. High resistance rates to Aminoglycosides (98\%) and Fluoroquinolones (99\%) has been reported by Qureshi et al, which is higher compared to our study. ${ }^{12}$ Ciprofloxacin, which was once proposed as an alternative therapy for MRSA infections, appears to be very ineffective in the present situation ${ }^{7-9}$ In general; elevated rates of multi drug resistance may emerge from diverse isolates of S.aureus under antimicrobial pressure or because of wide spread person-to-person transmission of multi drug resistant isolates. ${ }^{1}$

Despite being multidrug resistant, all the isolates were susceptible to Vancomycin using the disc diffusion technique. Looking to of the lower sensitivity of the disc diffusion method, use of a Vancomycin screen agar plate as well as a non-automated MIC method are mandatory to detect VISA/VRSA (Vancomycin-Intermediate/Resistant Staphylococcus aureus) isolates. All our MRSA isolates were also found to be sensitive to Linezolid which is the first oxazolidinone antibiotic approved for treatment of Gram-positive infections. If they are used judiciously, Vancomycin and Linezolid remain the mainstay for treating multi drug resistant MRSA infections.

To conclude, the prevalence of MRSA at our centre was very high and these were multi drug resistant. Looking to the nosocomial nature of these infections, a regular surveillance of hospital associated infections along with implementation of strict infection control measures and formulation of a definite antibiotic policy would aid in reducing the national prevalence of MRSA infections.

\section{REFERENCES:}

1. Kim HB, Jang HC, Nam HJ, et al. In vitro Activities of 28 antimicrobial agents against Staphylococcus aureus isolated from tertiary care hospitals in Korea: a Nationwide Survey. Antimicrob. Agents Chemother, 2004; 48 (4): 1124-27.

2. Jun IS, Tomoko F, Katsutoshi $\mathrm{S}$ et al.Prevalence of erythromycin, tetracycline \& aminoglycoside resistance genes in methicillin-resistant Staphylococcus Aureus in hospitals in Tokyo \& Kumamoto. Jpn J Infect Dis 2004: 57: 75-7.

3. Maple PAC, Hamilton-Miller JMT, Brumfitt W. Worldwide antibiotic resistance in methicillin resistant Staphylococcus aureus. Lancet 1989; 1:537-40.

4. Shanson DC. Antibiotic resistant Staphylococcus aureus. J. Hosp. Infect. 1981; 2: 11. 
5. Pavillard R., Harvey K., Douglas D., et al. Epidemic of hospital acquired infection due to methicillin resistant Staphylococcus aureus in major Victorian hospitals. Med. J. Aust. 1982 1:451-4.

6. Rajaduraipandi K, Mani KR, Panneerselvam K, et al. Prevalence and antimicrobial susceptibility pattern of methicillin resistant Staphylococcus aureus: a multicentre study. Indian J Med Microbiol. 2006; 24:34-8.

7. Majumder D, Sarma Bordoloi JN, Phukan AC, et al. Antimicrobial susceptibility pattern among methicillin resistant Staphylococcus isolates in Assam. Ind. J. Med. Microbiol. 2001; 19(3): 138-40.

8. Mehta A, Rodrigues C, Kumar R, et al. A pilot programme of MRSA surveillance in India (MRSA Surveillance Study group). Postgrad. Med. J. 1996; 42(1): 1-3.

9. Anupurba S, Sen MR, Nath G, et al. Prevalence of methicillin resistant Staphylococcus aureus in a tertiary referral hospital in eastern Uttar Pradesh. Ind. J. Med. Microbiol. 2003; 21(1): 49-51.

10. Verma S, Joshi S, Chitnis V, et al. Growing problem of Methicillin resistant Staphylococci: Indian Scenario. Ind. J. Med. Sci. 2000; 54(12): 535-40.

11. Mohanty S, Kapil A, Dhawan B, et al. Bacteriological and antimicrobial susceptibility profile of soft tissue infection from northern India. Ind. J. Med. Sci. 2004; 58(1): 10-15.

12. Qureshi AH, Rafi S, Qureshi SM et al . The current susceptibility patterns of Methicillin resistant Staphylococcus aureus to conventional anti Staphylococcus antimicrobials at Rawalpindi. Pak J Med Sci.2004; 20:361-4.

13. Sachdev D, Amladi S, Natraj G, et al. An outbreak of Methicillin resistant Staphylococcus aureus (MRSA) infection in dermatology indoor patients. Indian J. Dermatol. Venereal. Leprol. 2003; 69(6):377-80.

14. Assadullah S, Kakru DK, Thoker MA et al. Emergence of low level Vancomycin resistance in MRSA. Indian J Med Microbiol 2003; 21: 196-8

15. Baird D. Staphylococcus; Cluster-forming Gram-positive cocci. In: Mackie and Mac Cartney Practical Medical Microbiology 14th Ed. Collee JG, Fraser AG, Marmion BP, Simmons A, Eds. Edinburgh: Churchill Livingstone, 1996: 245-58.

16. NCCLS. Performance Standards for Antimicrobial Disk Susceptibility Testing; Approved Standard. - 7th Ed .NCCLS document M2-A7, 20: 2000.Wayne Pennsylvania.

17. NCCLS. Methods for Dilution Antimicrobial Susceptibility Tests for Bacteria That Grow Aerobically: Approved Standard - 6th Ed. NCCLS document M7-A6, 23: 2003. Wayne Pennsylvania. 
Table-1 Number and percentage distribution of MRSA from different clinical samples

\begin{tabular}{|lcc|}
\hline \multicolumn{1}{|c}{ Clinical Samples } & $\begin{array}{c}\text { Number and percentage of } \\
\text { S.aureus (n=202) }\end{array}$ & $\begin{array}{c}\text { Number and percentage of MRSA } \\
\text { (n=114) }\end{array}$ \\
Blood & $102(50.5 \%)$ & $58(50.88 \%)$ \\
Pus \& Wound swabs & $68(33.66 \%)$ & $37(32.46 \%)$ \\
Sputum / Throat swabs & $14(6.93 \%)$ & $07(06.14 \%)$ \\
Other body fluids & $18(8.91 \%)$ & $12(10.52 \%)$ \\
\hline
\end{tabular}

Figure-1: RESISTANCE PATTERN OF MRSA VIS MSSA ISOLATES

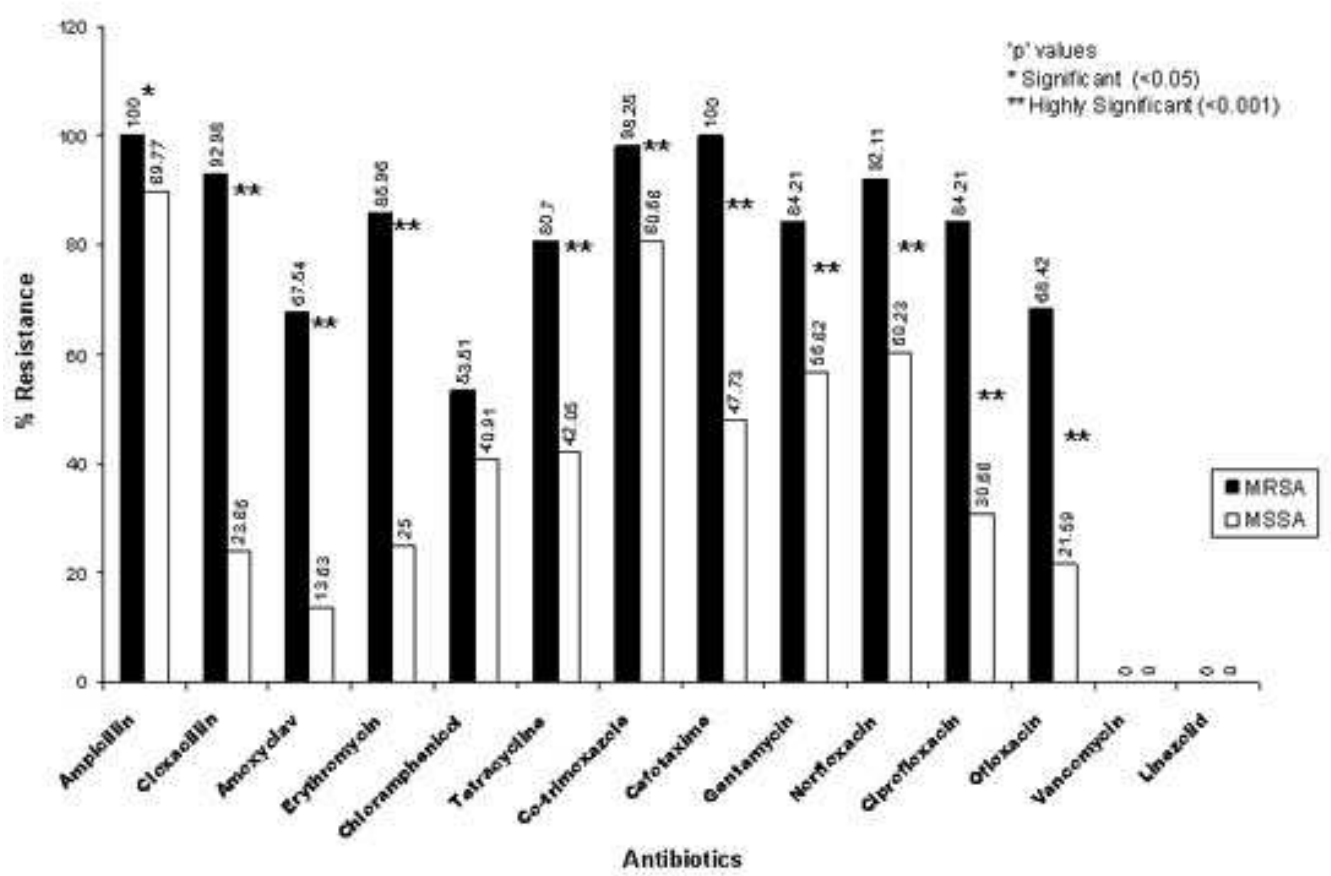

